\title{
XMM-Newton discovery of an X-ray filament in Coma
}

\author{
A. Finoguenov ${ }^{1}$, U. G. Briel ${ }^{1}$, and J. P. Henry ${ }^{2}$ \\ 1 Max-Planck-Institut für Extraterrestrische Physik, Giessenbachstraße, 85748 Garching, Germany \\ 2 Institute for Astronomy, University of Hawaii, 2680 Woodlawn Drive, Honolulu, Hawaii 96822, USA
}

Received 12 January 2003 / Accepted 12 August 2003

\begin{abstract}
XMM-Newton observations of the outskirts of the Coma cluster of galaxies confirm the existence of a soft X-ray excess claimed previously and show it comes from warm thermal emission. Our data provide a robust estimate of its temperature $(\sim 0.2 \mathrm{keV})$ and oxygen abundance ( 0.1 solar). Using a combination of XMM-Newton and ROSAT All-Sky Survey data, we rule out a Galactic origin of the soft X-ray emission. Associating this emission with a $20 \mathrm{Mpc}$ region in front of Coma, seen in the skewness of its galaxy velocity distribution, yields an estimate of the density of the warm gas of $\sim 50 f_{\text {baryon }} \rho_{\text {critical }}$, where $f_{\text {baryon }}$ is the baryon fraction and $\rho_{\text {critical }}$ is the critical density needed to halt the expansion of the universe. Our measurement of the gas mass associated with the warm emission strongly support its nonvirialized nature, suggesting that we are observing the warm-hot intergalactic medium (WHIM). Our measurements provide a direct estimate of the $\mathrm{O}, \mathrm{Ne}$ and $\mathrm{Fe}$ abundance of the WHIM. Differences with the reported Ne/O ratio for some OVI absorbers hints at a different origin of the OVI absorbers and the Coma filament. We argue that the Coma filament has likely been preheated, but at a substantially lower level compared to what is seen in the outskirts of groups. The thermodynamic state of the gas in the Coma filament reduces the star-formation rate in the embedded spiral galaxies, providing an explanation for the presence of passive spirals observed in this and other clusters.
\end{abstract}

Key words. galaxies: clusters: individual: Coma - cosmology: observations - intergalactic medium - large-scale structure of universe

\section{Introduction}

The total amount of baryons directly observed in the local Universe is only $\sim 35 \%$ of the value implied from primordial nucleosynthesis, with that observed at high-redshift (Fukugita et al. 1998, Table 3 excluding line $7^{\prime}$ ) and that observed within local closed box systems, such as clusters of galaxies (Briel et al. 1992). Numerical hydrodynamic simulations (e.g. Cen \& Ostriker 1999; Davé et al. 2001) indicate that about half of these missing baryons reside in a warm-hot intergalactic medium (WHIM). The temperature of the WHIM is expected to be between $10^{5}$ and $10^{7} \mathrm{~K}$ with an overdensity with respect to the mean of $\delta \sim 20$. Most of the WHIM baryons are expected to reside outside virialized regions in diffuse large-scale filaments.

Several surveys have been undertaken to find the WHIM in absorption. Tripp et al. (2000) have claimed a substantial fraction of the missing baryons in the form of OVI absorbers. However, their observations lack information on the ionization equilibrium as well as the $\mathrm{O}$ abundance required to link the abundance of OVI ions to underlying $\mathrm{O}$ mass and then to the amount of baryons. Chandra and XMM grating results on detection of OVII and OVIII absorption lines (Nicastro et al. 2002; Fang et al. 2002; Mathur et al. 2003; Fang et al. 2003; Rasmussen et al. 2003) allowed for the first time an estimate

Send offprint requests to: A. Finoguenov, e-mail: alexis@xray.mpe.mpg.de of the ionization state of the gas, suggesting that the OVI absorbers account for over $80 \%$ of the local baryons. Still, such claims rely on the assumed $\mathrm{O}$ abundance.

Searches for the WHIM in emission with ROSAT have found some association of soft X-rays with galactic filaments (Wang et al. 1997; Kull \& Böhringer 1999; Scharf et al. 2000; Zappacosta et al. 2002). Upper limits have been placed on emission by material connecting rich clusters of galaxies (Briel \& Henry 1995) using stacked analysis of ROSAT All-Sky Survey data. The corresponding limit on the emitting gas density is $\delta<375$, assuming a temperature of $1 \mathrm{keV}$ and an iron abundance of 0.3 solar. These observations may also be used to constrain the WHIM because most of it is expected to be in filaments connecting clusters.

The low filament densities implied by the weak absorption and soft X-ray emission, and expected from typical overdensities in simulations, complicates WHIM detection and study, unless the filament containing it is viewed along the line of sight. A combination of both the requirement that a filament be so viewed and the expectation that clusters are at the intersection of filaments, indicates that searches for WHIM near clusters of galaxies may prove fruitful. The soft X-ray excess above the harder intracluster medium (ICM) emission reported for some clusters (Lieu et al. 1996; Bonamente et al. 2002) may be signaling a fortunate orientation of a filament containing WHIM at those clusters. 
Table 1. Characteristics of the warm emission around Coma.

\begin{tabular}{cccrccccc}
\hline \hline $\begin{array}{c}\text { Coma } \\
\text { field }\end{array}$ & $\begin{array}{c}\text { Exposure } \\
\mathrm{ksec}\end{array}$ & $\begin{array}{c}k T_{\text {Coma }} \\
\mathrm{keV}\end{array}$ & $\begin{array}{r}k T_{\text {warm }} \\
\mathrm{keV}\end{array}$ & $\begin{array}{c}Z / Z_{\odot}^{b} \\
\delta_{\text {crit }}^{a}\end{array}$ & $\begin{array}{c}0.5-2^{c} \\
\mathrm{keV}\end{array}$ & \multicolumn{2}{c}{$\mathrm{O}^{c}$} \\
\hline 0 & 14.7 & $15 \pm 6$ & $0.19 \pm 0.01$ & $0.06 \pm 0.02$ & $67 \pm 7$ & $10.3 \pm 3.4$ & $3.9 \pm 1.4$ & $1.6 \pm 0.6$ \\
3 & 11.9 & $5.8 \pm 0.9$ & $0.19 \pm 0.02$ & $0.04 \pm 0.02$ & $76 \pm 12$ & $8.6 \pm 4.3$ & $3.0 \pm 1.6$ & $1.2 \pm 0.6$ \\
7 & 21.2 & $10 \pm 3$ & $0.22 \pm 0.02$ & $0.07 \pm 0.03$ & $45 \pm 18$ & $4.7 \pm 2.0$ & $1.2 \pm 0.7$ & $0.8 \pm 0.5$ \\
11 & 13.3 & $16 \pm 2$ & $0.24 \pm 0.01$ & $0.09 \pm 0.01$ & $71 \pm 5$ & $27 \pm 3.0$ & $5.4 \pm 0.7$ & $5.2 \pm 0.7$ \\
13 & 22.4 & $3.1 \pm 0.5$ & $0.17 \pm 0.01$ & $0.12 \pm 0.04$ & $65 \pm 7$ & $8.7 \pm 2.9$ & $4.7 \pm 1.7$ & $1.2 \pm 0.4$ \\
\hline
\end{tabular}

a Filament overdensity relative to the critical, $\left(\rho / \rho_{\text {crit }}-1\right)$, estimated by assuming a $90 \mathrm{Mpc}$ distance, projected length of $20 \mathrm{Mpc}$, area of the extraction regions in Fig. 1 and $n_{\mathrm{e}}=8 \times 10^{-7} \mathrm{~cm}^{-3}$ in correspondence to $\rho_{\text {crit }}$ for $h=0.7$.

${ }^{b}$ Assuming a solar abundance ratio.

c Flux, $10^{-7}$ photons $\mathrm{cm}^{-2} \mathrm{~s}^{-1} \operatorname{arcmin}^{-2}$.

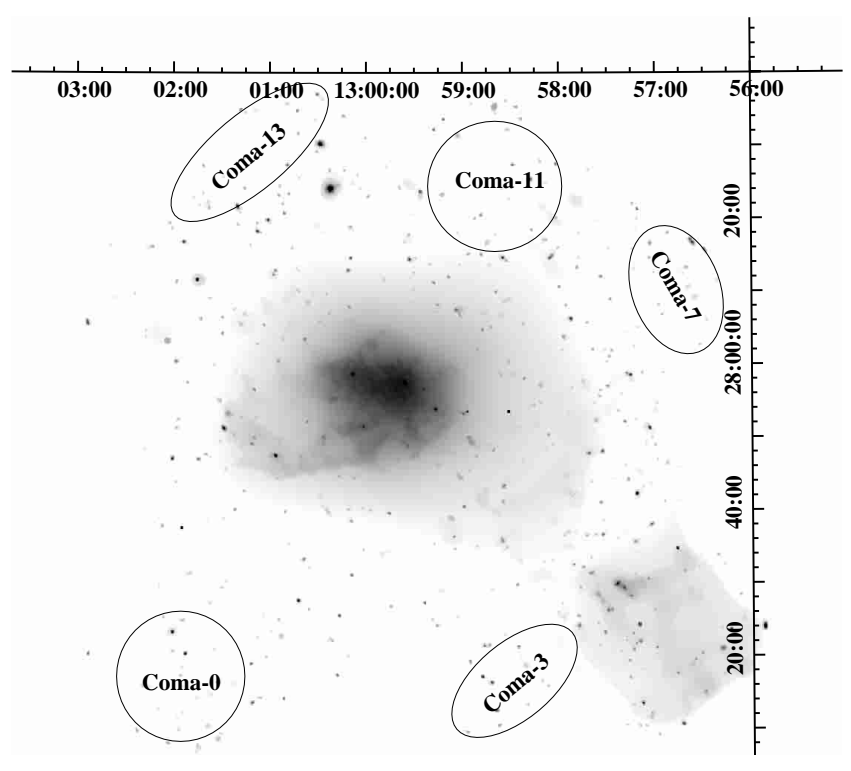

Fig. 1. An image of the Coma cluster in the $0.5-2 \mathrm{keV}$ band, showing the positions of the spectral extraction regions (solid ellipses) with names according to the XMM-Newton survey notation. The coordinate grid marks RA, Dec (J2000.0).

In this paper we carry out an X-ray spectroscopic study of the outskirts of the Coma cluster, which has a soft excess, in order to search for and characterize any WHIM at this location. Our observations are from the mosaic made by XMMNewton. Kaastra et al. (2003) report a similar study of the center of the Coma cluster also using data from the XMM mosaic. Not knowing the relative spatial distributions of the WHIM and ICM, it is difficult to predict whether observing the center or outskirts of a cluster will maximize the contrast of any warm component relative to the dominant hot component. However both studies do detect a warm component. We adopt a Coma cluster redshift of $0.023, H_{\mathrm{o}}=70 \mathrm{~km} \mathrm{~s}^{-1} \mathrm{Mpc}^{-1}$, and quote errorbars at the $68 \%$ confidence level. One degree corresponds to $1.67 \mathrm{Mpc}$.

\section{Observations}

The initial results of the XMM-Newton (Jansen et al. 2001) performance verification observations of the Coma cluster are reported in Briel et al. (2001), Arnaud et al. (2001) and Neumann et al. (2001). In addition to the observations reported there, three additional observations have been carried out, completing the planned survey of the Coma cluster. We have renamed the Coma-bkgd field from Briel et al. (2001) Coma-0 in order to avoid confusion with the additional background pointings required to analyze these data.

Since the Coma cluster is a large diffuse source, estimating the background is non-trivial. In general we follow the method of Lumb et al. (2002). The observations reported here are performed with the EPIC pn detector (Strüder et al. 2001) using its medium filter. This configuration leads to a different detector count rate from the cosmic (CXB) and Milky Way $\mathrm{X}$-ray backgrounds in the soft band, compared to the usual thin filter. The Milky Way background is further composed of the Local Bubble and halo components. We used a 100 ks observation of the point source APM 08279+5255 (PI G. Hasinger), performed with the same (medium) filter, to measure these background components and a filter wheel closed observation to measure the internal or particle background. We used the APEC plasma code (Smith et al. 2001) to fit the Milky Way background components and a power-law $(\Gamma=1.45)$ to fit the CXB. The element abundance in the APEC code was fixed to solar and temperature was set to $0.07 \mathrm{keV}$ for the Local Bubble and left as a free parameter for the halo, yielding a $0.22 \mathrm{keV}$ best-fit value. To ensure the compatibility of the CXB, we excised from the spectral extraction region the APM quasar and the X-ray sources identified with Coma galaxies (which otherwise increase the apparent CXB flux by 10\%). Since there is a mismatch in the $N_{\mathrm{H}}$ toward the Coma and the APM fields, we use the same spectral shape and normalization of the halo and CXB components, but changed their $N_{\mathrm{H}}$ from $3.9 \pm 0.3 \times 10^{20} \mathrm{~cm}^{-2}$ (APM) to $9 \times 10^{19} \mathrm{~cm}^{-2}$ (Coma). The Local Bubble is not subject to this absorption. We also checked that the derived results on the soft X-ray emission from Coma do not depend on the possible large-scale variation in the intensity of the Local Bubble component. 
For the spectral analysis we use the $0.4-6 \mathrm{keV}$ range and for the estimate of the detector background the $9.5-15 \mathrm{keV}$ range. We do not use the energies below $0.4 \mathrm{keV}$, to avoid a complicated treatment of detector background associated with the electronic noise (Lumb et al. 2002). This precludes us from studying the very soft emission. Although, we could potentially use the energies up to $10 \mathrm{keV}$ in the analysis, we are able to sufficiently constrain the hot component of the Coma cluster already without using these energy bands, where removal of the detector background becomes critical.

The vignetting correction is performed taking the source extent and a recent pn telescope (XRT3) vignetting calibration (Lumb et al. 2003) into account, which is mostly important for the absolute flux determination, given our choice of the energy range. Systematic uncertainty of the flux determination is $4 \%$ for both pn and MOS.

In addition to a thorough evaluation of the background, we further reduce the systematics of our study by using the results of recent calibration efforts of the pn team of MPE on soft energy response of the pn detector (Haberl et al. 2002, XMMSAS-5.4 release). Furthermore, to improve on the detection statistics of the warm emission and to reduce the systematic effects of subtraction of the cluster emission, we concentrate on the outskirts of the Coma cluster.

We investigated some systematic errors in our analysis by determining the effects of using experimental software or nonstandard values of some parameters. We do not use these modifications in our analysis, however. The effect of pn energy scale calibration was evaluated by comparing the results of the standard analysis with experimental software that corrects the zero point of the energy scale for each pixel of the pn. We found some subtle differences, e.g. a higher best-fit temperature by $0.03 \mathrm{keV}$, which is larger than the statistical error quoted in Table 1. However, the ratio of $\mathrm{O}$ to Ne was still found to be solar or larger (see Sect. 5). Inclusion of ROSAT All-Sky Survey (RASS) data into the analysis of the background allowed us to constrain better the Local Bubble component, e.g. a temperature of $0.10 \pm 0.01 \mathrm{keV}$ was found but with a somewhat lower luminosity. Using these values instead of the standard ones quoted above had no effect on our results.

In Fig. 1 we show the location of the spectral extracting areas. We select regions $\sim 40^{\prime \prime}$ from the Coma center to the NorthWest, North, North-East, South and South-East. The SouthWest direction is complicated by the presence of the infalling subcluster (NGC 4839), while some other pointings were affected by background flares, which after screening lead to insufficient exposures.

\section{Results}

Our major results are shown in Fig. 2 and listed in Table 1. We find that the soft excess in Coma on spatial scales of $30^{\prime}-50^{\prime}$ is characterized by thermal plasma emission, because of the clear detection of $\mathrm{O}$ lines, with a characteristic temperature of $\sim 0.2 \mathrm{keV}$. Bonamente et al. (2003) found the same temperature characterizing the ROSAT PSPC observation of the Coma soft excess. The highest temperature of this component corresponds to the hot spot in Coma found in ASCA observations
(Donnelly et al. 1999), and identified as an accreting zone of the Coma cluster.

We have performed several internal checks of our results. First, Fig. 3 shows the confirmation of our findings for the Coma-11 field by analysis of the XMM-Newton MOS1 data. The medium filter was also used for the MOS during these observations. For this analysis we have done a simple background analysis that uses the flux in the MOS1 field for the same instrument settings and background conditions but a different $N_{\mathrm{H}}$. Since the MOS temperature of the hot component is consistent with that determined by the pn, we fix the temperature of that component to its pn value in the MOS spectral analysis. The MOS fit then agrees with the pn results for the intensities of the hot and warm components and for the temperature and element abundance for the warm component.

Second, to entirely exclude the effect of the instrumental or particle background, we have attempted an in-field background subtraction for the pn. Using the difference in the spatial distribution of the Coma hot and detector background components within the Coma-11 field, we were able to remove both of them using data only from the Coma-11 field. In particular, the region south of the pointing center contains more of the cluster emission relative to the detector background and vice-versa for a region north of the pointing center. Since the spectrum of the $\mathrm{CXB}$ is similar to that of Coma hot emission (see Fig. 2), it could be removed by over-subtracting the Coma hot flux. We exclude energies corresponding to the detector emission lines from the comparison, as their distribution is different from that of the continuum (e.g. Lumb et al. 2002). The resulting spectrum is displayed in Fig. 2 and could be fitted with a single MEKAL model with $0.22 \pm 0.01 \mathrm{keV}$ temperature and a factor of 3 higher intensity than the Milky Way halo plus Local Bubble emission. Third, we have tried several compilations of the XMM background (Lumb et al. 2002; Read 2003) and found that the soft component in the Coma emission is clearly seen using any of them.

At the moment, it is difficult from the $\mathrm{O}$ line redshift alone to decide whether this emission is Galactic or extragalactic. However, some apparent differences with the Galactic emission are already noticeable. The soft component is centered on Coma and has an amplitude exceeding the variation of the underlying Galactic emission (Bonamente et al. 2003). An $\mathrm{O}$ abundance of 0.1 times solar is much lower than the Galactic value of one solar (Markevitch et al. 2002; Freyberg \& Breitschwerdt 2002). The temperature variations of the Coma warm component are not seen in the quiet zones of Galactic emission at high latitudes. The observed warm component exceeds the level of the Milky Way halo plus Local Bubble continuum (at $0.57 \mathrm{keV}$ ) emission by a factor of 3 . Also the strength of the observed $\mathrm{O}$ lines are a factor of three larger than the corresponding values for the Galactic emission, and to significantly change the derived $\mathrm{O}$ abundance of the Coma warm emission a possible variation in the metallicity for the Galactic emission should be by a factor of $\sim 3$, which is not observed (Freyberg \& Breitschwerdt 2002). As we will demonstrate below, ROSAT PSPC data strongly constrain the contribution of the low-temperature Galactic components to the observed $\mathrm{O}$ line flux. 


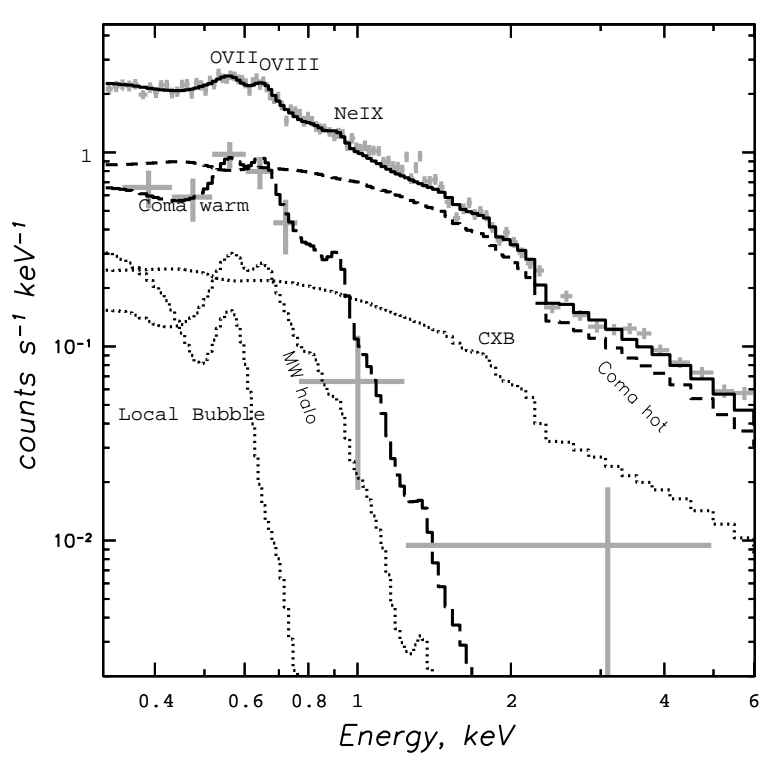

Fig. 2. Detailed decomposition of the pn spectrum (small grey crosses) in the Coma-11 field into foreground and background components, obtained from the analysis of the APM field, plus hot and warm emission from the Coma cluster. Large crosses are the result of the in-field subtraction of Coma emission and detector background, possible due to differences in the spatial distributions between the Coma warm, Coma hot and detector background components. MW halo means Milky Way halo and CXB means cosmic X-ray background.

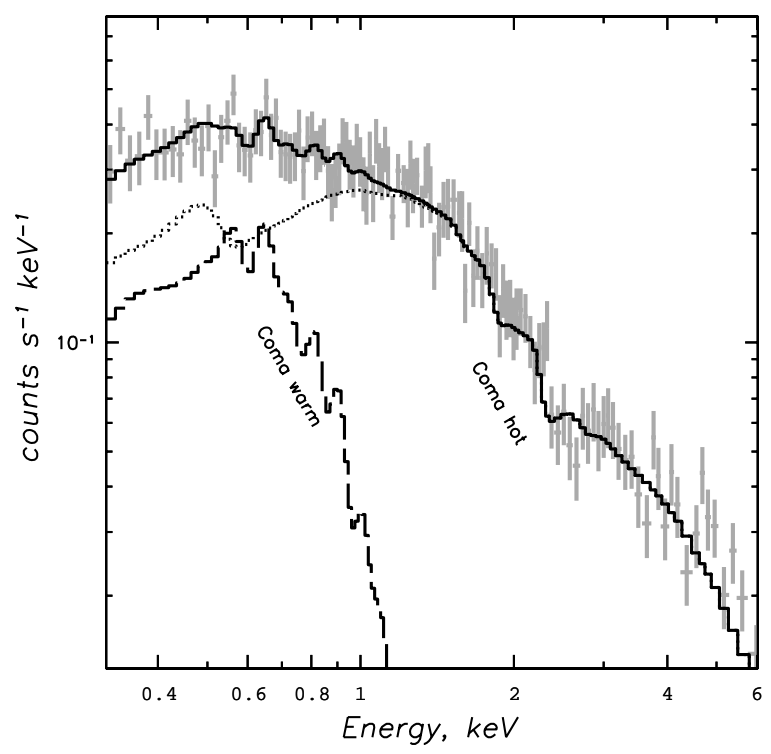

Fig. 3. MOS 1 APM field background subtracted spectrum of the Coma-11 field. Detailed modeling, accounting for the differences in $N_{\mathrm{H}}$ was not performed, but the difference is small, given the lower sensitivity of MOS CCDs at low energies compared to the pn.

\section{Comparison with the ROSAT All-Sky Survey}

We use the RASS to provide an external check of our results. We note, however, that the spatial and spectral resolution and energy band of the ROSAT detectors are significantly different from those on XMM so care needs to be taken when performing this check.

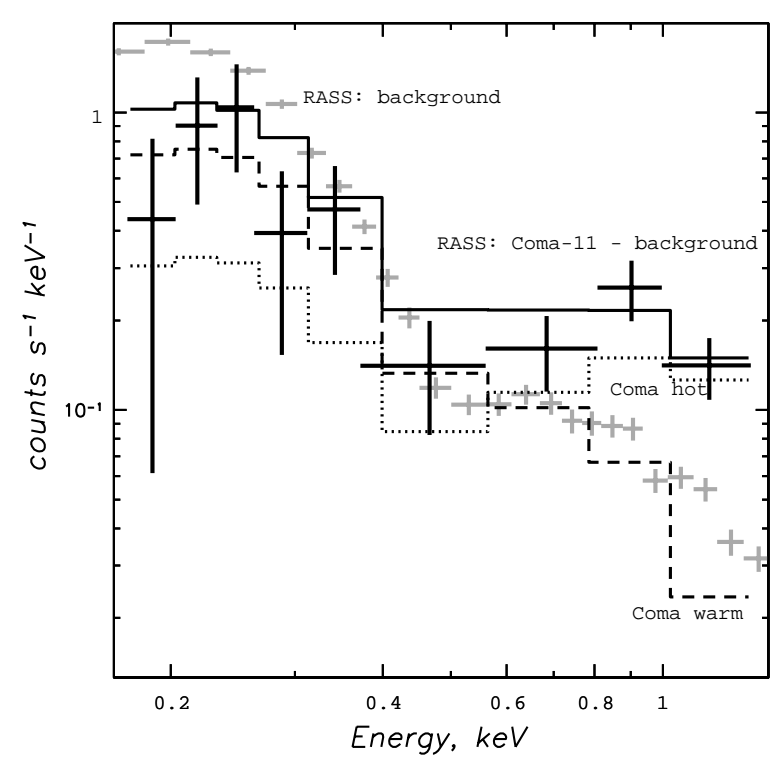

Fig. 4. ROSAT All-Sky Survey spectrum of Coma-11. Grey crosses show the background spectrum $2^{\circ}$ north of the Coma center. Black crosses show the background subtracted spectrum extracted at the position of the Coma-11 field. Lines show the best fit spectrum from the analysis of pn data folded through the ROSAT PSPC with no adjustment to the pn best fit. Dashed line is the warm component, dotted line is the hot component and the solid line is the sum. The best fit model to the pn data provides a statistically acceptable fit to the RASS data $\left(\chi^{2}=9.8\right.$ per 9 d.o.f.).

We first compare the fluxes implied by our analysis to those in the RASS data as presented in Snowden et al. (1997). We choose the $0.5-0.7 \mathrm{keV}$ band (ROSAT R4 band), corresponding to position of the $\mathrm{O}$ lines and the $1.3-2.0 \mathrm{keV}$ band (R7), where the relative contribution of the hot component is the largest. We make the comparison for two fields: Coma- 11 and a field $4^{\circ}$ North of Coma, which we call Sky Background. Several effects should be taken into account to provide a proper comparison between the XMM-Newton and ROSAT data. First, the X-ray mirrors of XMM-Newton have a 7\% efficiency to stray-light (Lumb et al. 2002) with uncertainty in the energy dependence of $2 \%$. This results in higher fluxes seen by the XMM detectors near bright objects and may lead to object-specific flux variations. We use the RASS data to estimate the effect of stray-light pollution in the background and Coma fields by assuming uniform azimuthal and radial dependence of the stray-light coming from XMM off-axis angles of $0.4^{\circ}-1.4^{\circ}$. This component should be subtracted from the XMM fluxes in the comparison. Second, the RASS maps of the diffuse X-ray background have point sources removed, which reduces the intensity of the background. We use the limits of Snowden et al. (1997) and the $\log N-\log S$ of Hasinger et al. (2001) to estimate this removed flux. This component should be added to the RASS flux in the comparison. Third, RASS data on the sky background on the scales of the XMM field of view have rather large statistical uncertainties and some averaging should be carried out in order to provide a meaningful comparison. Fourth, the R4 images exhibit variations larger than the statistical errors, so we quote the errors corresponding to the systematic variations. Fifth, in the analysis of XMM spectra a separate power-law component 
Table 2. Comparison between XMM-Newton Predictions of the ROSAT Surface Brightness from Observations (Coma-11) or Background Model (Sky Back) and Observed ROSAT All-Sky Survey Data.

\begin{tabular}{ccccc}
\hline \hline $\begin{array}{c}\text { Field } \\
\text { name }\end{array}$ & \multicolumn{2}{c}{ XMM-Newton pn } \\
predicted & \multicolumn{2}{c}{$\begin{array}{c}\text { RASS } \\
\text { stray-light } \\
\text { 10 }\end{array}$} & $\begin{array}{c}\text { PSPC-C counts } \mathrm{s}^{-1} \\
\text { arcmin }^{-2}\end{array}$ \\
\hline \multicolumn{5}{c}{$0.5-0.7 \mathrm{keV}$} \\
Coma-11 & $2.7 \pm 0.1$ & 0.10 & $2.0 \pm 0.5$ & 0.2 \\
Sky Back & $0.66 \pm 0.08$ & 0.05 & $0.5 \pm 0.1$ & 0.2 \\
\multicolumn{5}{c}{$1.3-2.0 \mathrm{keV}$} \\
Coma-11 & $3.2 \pm 0.1$ & 0.14 & $3.2 \pm 0.7$ & 0.2 \\
Sky Back & $0.56 \pm 0.04$ & 0.05 & $0.4 \pm 0.02$ & 0.2 \\
\hline
\end{tabular}

was introduced to remove the increased detector background due to soft protons (De Luca \& Molendi 2002). Studies of this component by several groups (Katayama et al. 2002; Snowden \& Molendi 2003, private communications) show that its intensity could be determined by the spectrum at high energies $(10-15 \mathrm{keV})$ where the mirrors have no collecting power for $\mathrm{X}$-rays, while the instrumental background originates from energy losses of particles on the detector. From the comparison of the XMM predicted and the RASS observed intensities of the Sky Background presented in Table 2 it is clear that our background model is applicable for the Coma cluster. Our results for Coma-11 are also in agreement with the RASS flux, although the later has a rather large uncertainty.

The pixel size in the Snowden maps is rather large (12'), so we have performed a second analysis by extracting a spectrum from the raw RASS data at the exact location of the Coma-11 field, a circle of $8^{\prime}$ radius centered on $\alpha=194.66521^{\circ}, \delta=$ $28.406924^{\circ}$. We have extracted a background spectrum using a $0.6^{\circ} \times 2^{\circ}$ region (long axis perpendicular to radius vector from the cluster center) $2^{\circ}$ North of the cluster. We display the results in Fig. 4. We find that our pn model provides an acceptable fit to the RASS data with no adjustable parameters $\left(\chi^{2}=9.8\right.$ per 9 degrees of freedom).

Figure 4 also shows that a simple comparison of the countrates between the two instruments is not straightforward, particularly at energies below $0.5 \mathrm{keV}$. The ROSAT background is very high in this energy range compared to the pn. This is caused by two differences between the instruments. At an energy of $0.25 \mathrm{keV}$ the effective area of the ROSAT PSPC is about a factor of two higher than the XMM pn $\left(\sim 175 \mathrm{~cm}^{-2}\right.$ vs. $\sim 80 \mathrm{~cm}^{-2}$ ) while the energy resolution of the PSPC is about a factor of two worse than the pn ( 1 vs. $\sim 2)$ (Snowden et al. 1997 for the PSPC, XMM Users Handbook for the pn). At $0.25 \mathrm{keV}$ all components are dominated by the Local Bubble. The poor energy resolution of the ROSAT PSPC redistributes the larger number of counts from this component to higher energies. On the other hand, since the XMM-Newton Coma observations were made with the medium filter, most of the counts at lower energies are actually redistributed from higher energies. This is why our results are not so sensitive to the model of the Local Bubble. The combination of these two effects produces substantial differences in the ratio of the Coma counts to the background counts below $0.5 \mathrm{keV}$ in the two instruments and a proper comparison requires detailed spectral modeling as Fig. 4 shows.

The comparison of Figs. 2 and 4 reinforces our previous analysis using the Snowden maps, but with a finer spatial and spectral resolution. For example, the intensity of the total background continuum at $0.57 \mathrm{keV}$ in both figures is about equal to the Coma warm continuum. However, the unlimited field of view of the RASS allows a direct measurement of the background in Fig. 4 compared to a model of the background in Fig. 2. Thus we conclude that an incorrect background model producing the observed Coma Warm component is ruled out.

Our analysis of the ROSAT data can also be used to set limits on the neutral gas fraction in a filament. From Table 1, the HII column is about $10^{21} \mathrm{~cm}^{-2}$. From Fig. 4 the limit on the column of $\mathrm{HI}$ above the Galactic value is $<10^{20} \mathrm{~cm}^{-2}$. Hence, the $\mathrm{HI}$ fraction is less than $10 \%$. Marginal indication of such absorption is present in Fig. 4, as the data points are slightly below the model prediction.

\section{Interpretation}

In the rest of this paper we present an interpretation assuming an extragalactic origin of the warm component. With the CCDtype spectrometry we can place limits on the possible redshifts of the warm component, which for the Coma-11 field is determined as $0.007 \pm 0.004 \pm 0.015$ (best-fit, statistical and systematic error). Any extragalactic interpretation should therefore concentrate on the large-scale structure in front of the Coma cluster since the allowed redshift is $0.000-0.026$ compared to the Coma value of 0.023 . We have carried out an analysis of the CfA2 galaxy catalog (Huchra et al. 1995) towards the Coma cluster, excising the South-West quadrant, where strong influence of the infalling subcluster NGC 4839 on the velocity distribution has been suggested (Colles \& Dunn 1996). Figure 5 illustrates our result, indicating a significant galaxy concentration in front of the Coma cluster, with velocities lying in the $4500-6000 \mathrm{~km} \mathrm{~s}^{-1}$ range. The sky density of the infall zone is shown in the insert of Fig. 5. It was constructed from the number of galaxies in the $4500-6000 \mathrm{~km} \mathrm{~s}^{-1}$ velocity range minus the number of galaxies in the $8500-10000 \mathrm{~km} \mathrm{~s}^{-1}$ bin, corresponding to a similar difference in the velocity dispersion from the cluster mean. By excising the galaxies at velocities lower than $6000 \mathrm{~km} \mathrm{~s}^{-1}$, we recover a projected velocity dispersion of $\sim 750 \mathrm{~km} \mathrm{~s}^{-1}$. Using the $M-\sigma$ relation of Finoguenov et al. (2001), we find that such velocity dispersion is more in accordance with mass of the Coma cluster. The excess of galaxies exhibits a flat behavior within 0.8 degrees $(1.3 \mathrm{Mpc})$, followed by a decline by a factor of 5 within 1.7 degrees $(2.8 \mathrm{Mpc})$ and a subsequent drop by two orders of magnitude within 10 degrees $(16.7 \mathrm{Mpc})$ The soft emission from the Coma is detected to a 2.6 Mpc distance from the center (Bonamente et al. 2003) in a remarkable correspondence to the galaxy filament.

In the absence of the finger-of-god effect (decoupling of galaxies from the Hubble flow), validated below by our conclusion on the filamentary origin of this galaxy concentration, the infall zone is characterized by a $20 \mathrm{Mpc}$ projected length. This length assumption affects the density estimates, while the $\mathrm{O}$ abundance is only based on the assumption of collisional 
equilibrium. We have verified the later assumption by studying the ionization curves for OVII and OVIII presented in Mathur et al. (2003). We have concluded that the assumption of pure collisional ionization is valid for our data, since $n_{\mathrm{e}}>10^{-5} \mathrm{~cm}^{-3}$ and $T>2 \times 10^{6} \mathrm{~K}(0.17 \mathrm{keV})$. An advantage of our measurement is that we also determine the temperature by the continuum. Lower temperatures, which at densities near $10^{-5} \mathrm{~cm}^{-3}$ result in similar line ratios for OVII and OVIII, fail to produce the observed (H-e and He-e) bremsstrahlung flux at $0.7-1 \mathrm{keV}$.

An important question we want to answer is whether the detected emission originates from a group or from a filament. We cannot decide from the electron density of the structure $\left(\sim 50\left(\mu_{\mathrm{e}} / \mu_{\mathrm{p}}\right) f_{\text {baryon }} \rho_{\text {crit }}\right)$, as it suits both (for the low metallicity of filament we take $\mu_{\mathrm{e}}=1.1, \mu_{\mathrm{p}}=1.2$ and $f_{\text {baryon }}=0.16$ to correspond to the first WMAP results in Spergel et al. 2003). However, the implied mass of the structure is comparable to the mass of the Coma cluster (see also Bonamente et al. 2003). On the other hand the temperature of the emission is $\sim 0.2 \mathrm{keV}$, almost two orders of magnitude lower than that of the Coma cluster. It takes a few hundred groups with virial temperature of $0.2 \mathrm{keV}$ to make up a mass of the structure, which leads to an overlapping virial radii if we are to fit them into the given volume of the structure. Thus we conclude that the observed structure is a filament.

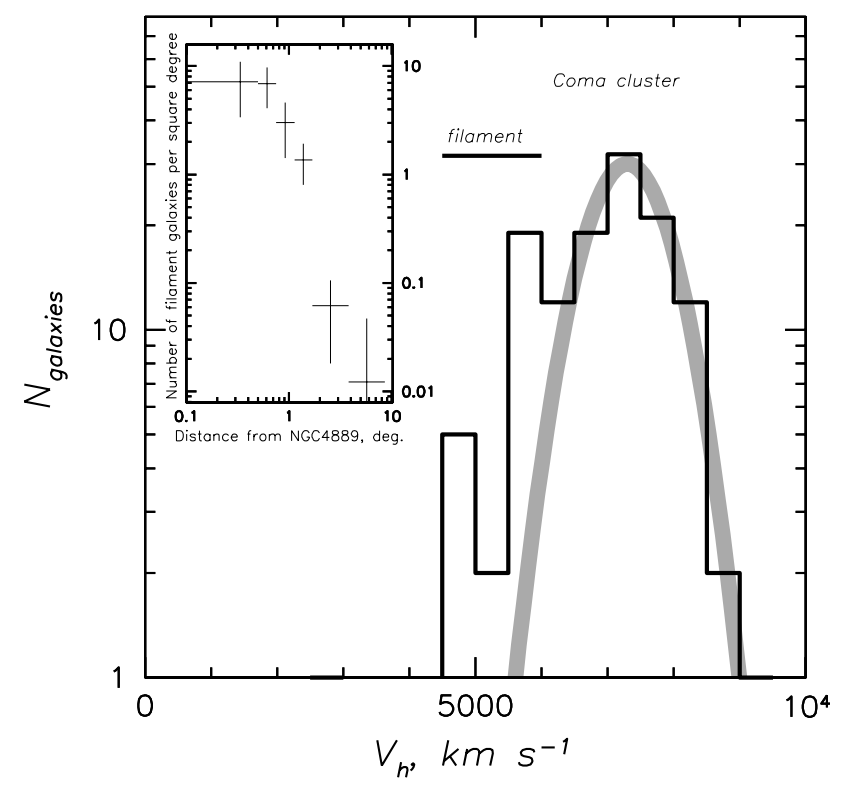

Fig. 5. Galaxy distribution in the direction to the Coma cluster from CfA2 survey. Central 2 degrees in radius field centered on NGC 4889 are shown, excluding the 60 degrees cone in the South-West direction from the center, to avoid the effect of the NGC 4839 subcluster. An excess of galaxies over the Gaussian approximation of the velocity dispersion of the Coma cluster is seen in the $4500-6000 \mathrm{~km} \mathrm{~s}^{-1}$ velocity range. The insert shows the spatial distribution of the excess galaxies in the $4500-6000 \mathrm{~km} \mathrm{~s}^{-1}$ velocity bin over the galaxies in the $8500-10000 \mathrm{~km} \mathrm{~s}^{-1}$ velocity bin, this time out to 10 degrees.

For the Coma-11 field, where the statistics are the highest, we investigated the effect of relaxing the assumption of solar abundance ratios. We note that an assumption for $\mathrm{C}$ abundance is important for overall fitting and a solar $\mathrm{C}$ value would affect our results. When left free, however, the $\mathrm{C}$ abundance tends to go to zero. Also, there is no systematic dependence of our results on the assumed $\mathrm{C}$ abundance as long as the $\mathrm{C} / \mathrm{O}$ ratio is solar or less, which is the correct assumption from the point of view of chemical enrichment schemes and observations of metal-poor stars in our Galaxy. Significant abundance measurements are: $\mathrm{O} / \mathrm{O}_{\odot}=0.14 \pm 0.02, \mathrm{Ne} / \mathrm{Ne}_{\odot}=0.14 \pm 0.06$, $\mathrm{Fe} / \mathrm{Fe}_{\odot}=0.04_{-0.01}^{+0.03}$ (assuming $\mathrm{Fe}_{\odot} / \mathrm{H}=4.26 \times 10^{-5}$ by number), indicating that $\mathrm{Fe}$ is underabundant by a factor of three in respect to the solar $\mathrm{Fe} / \mathrm{O}$ ratio, implying a dominant contribution of SN II to Fe enrichment. Element abundances for the hot emission obtained from our XMM data on the center of Coma are $\mathrm{Mg} / \mathrm{Mg}_{\odot}=0.36 \pm 0.12, \mathrm{Si} / \mathrm{Si}_{\odot}=0.45 \pm 0.08$, $\mathrm{S} / \mathrm{S}_{\odot}=0.01 \pm 0.15, \mathrm{Fe} / \mathrm{Fe}_{\odot}=0.20 \pm 0.03$, also suggesting prevalence of SN II in Fe enrichment, although Fe abundance is a factor of 5 higher compared to the filament. The filament $\mathrm{Ne} / \mathrm{O}$ ratio reveals a subtle difference with the OVI absorbers. Nicastro et al. (2002) reports $\mathrm{Ne} / \mathrm{O}=2$ times solar for their $\mathrm{X}$-ray absorption observations of material associated with the OVI absorbers. We observe a lower (solar) ratio at 95\% confidence. Lower ratios seem to be a characteristic of enrichment at $z>2$ (Finoguenov et al. 2003b), while simulations suggest that the WHIM originates at $z<1$ (Cen \& Ostriker 1999).

While we would rather have more observational evidence on the dispersion of $\mathrm{O}$ to alpha element ratios at different sites, we believe that this is a major source of systematics in interpreting the element abundance of X-ray filaments as a universal value. To illustrate the point, we calculate the density of baryons traced by the OVI absorbers under two sets of assumptions. Scaling the original value of $\Omega_{\mathrm{b}}(\mathrm{OVI})=0.0043 h_{70}^{-1}$ of Tripp et al. (2000) for ionization equilibrium implied by measurements of Mathur et al. (2003) and using the measurements of the $\mathrm{O}$ abundance reported here yields:

$\Omega_{\mathrm{b}}^{\mathrm{OVI}}=0.020 h_{70}^{-1}\left(\frac{0.14}{10^{[\mathrm{O} / \mathrm{H}]}}\right)\left(\frac{\left\langle[f(\mathrm{OVI})]^{-1}\right\rangle}{32}\right)$.

The formal errorbar is 0.006 , mostly from the uncertainty in the estimate for ionization. If, on the other hand, our measurements of $\mathrm{Ne}$ abundance are used with the $\mathrm{Ne} / \mathrm{O}$ ratio for OVI absorbers from Nicastro et al. (2002) then,

$\Omega_{\mathrm{b}}^{\mathrm{OVI}}=0.040 h_{70}^{-1}\left(\frac{0.14}{10^{[\mathrm{Ne} / \mathrm{H}]}} \frac{10^{[\mathrm{Ne} / \mathrm{O}]}}{2}\right)\left(\frac{\left\langle[f(\mathrm{OVI})]^{-1}\right\rangle}{32}\right)$.

Therefore the second set of assumptions must be invalid since the total baryon density is $\Omega_{\mathrm{b}}^{\text {total }}=0.039$, leaving no room for other major components of local baryons such as $\mathrm{Ly}_{\alpha}$ absorbers $(0.012 \pm 0.002)$ and stars and clusters of galaxies ( 0.006; e.g., Finoguenov et al. 2003b and references therein). O depletion onto dust grains in the OVI absorbers, as suggested by Nicastro et al. (2002) as an explanation of the high $\mathrm{Ne} / \mathrm{O}$ ratio, does not explain the unacceptably high baryon abundance implied by Eq. (2), since the solar Ne/O ratio in our observations may be simply explained by dust sputtering.

The observational determination of scaling relations between X-ray properties, such as luminosity $L_{X}$, gas temperature $T$, and entropy is crucial in establishing the physical properties of the ICM. The slopes of the $L_{\mathrm{X}}-T$ and 
mass-temperature relations (e.g. Markevitch 1998; Finoguenov et al. 2001) and the gas entropy level (e.g. Ponman et al. 1999; Finoguenov et al. 2002) are all at variance with model predictions based on pure gravitational heating (Kaiser 1986) and require the introduction of extra physics to describe the thermodynamics of the ICM (e.g. Evrard \& Henry 1991; Kaiser 1991). An often discussed piece of extra physics is preheating of the baryons before they accrete onto the cluster. Most baryons that accrete onto clusters are thought to come from filaments, so we now have an opportunity to compare the thermodynamic properties of the filament gas with that of group and cluster gas. The entropy of the X-ray emitting filament is $150 \mathrm{keV} \mathrm{cm}$, which could be reproduced by heating while falling onto a filament (e.g. Cen \& Ostriker 1999). In fact, estimating the expected Mach number of the accretion shock when the filament enters the Coma cluster yields a value of 4 , similar to predictions of simulations by Miniati et al. (2000). Regardless of the origin of the entropy of the filamentary gas, its entropy is much smaller than the $400 \mathrm{keV} \mathrm{cm}^{2}$ implied by ASCA observations of the outskirts of groups (Finoguenov et al. 2002), ruling out a universal preheating value. We note that the energy of the gas $\left(\frac{3}{2} k T=0.36 \pm 0.02 \mathrm{keV} /\right.$ particle $)$ is similar, although higher than the $\mathrm{SNe}$ energy associated with enrichment of gas to the observed $\mathrm{O}$ abundance $(0.22 \pm 0.03 \mathrm{keV} /$ particle $)$, so heating by galactic winds is not ruled out.

Although, the temperature of the filament is presently a factor of 40 lower than the Coma virial temperature, as it falls into the cluster it will be shock heated, and its final adiabat will be higher than in the self-similar case (Dos Santos \& Doré 2002; Ponman et al. 2003). Since the mass of the filament is comparable to that of the Coma cluster, the combined object will also deviate from cluster scaling relations. Observations indicate that the gas-to-dark matter distribution will not be affected (Sanderson et al. 2003), but the temperature is higher for a given mass (Finoguenov et al. 2001). This process is probably not universal, since some groups of galaxies have shallow gas profiles, thus possibly pointing to adiabatic compression rather than shock heating of the preheated gas, as proposed by Tozzi \& Norman (2001).

If the structures reported here are only associated with large clusters of galaxies, their contribution to the baryon budget is negligible $(0.1-1 \%)$. Of greater importance is to investigate the accreting environment of the massive groups and poor clusters that have a significant entry in the baryon budget $(6 \%)$. However, early enrichment epoch, suggested by low Ne/O ratio, makes X-ray filaments a substantial entry in the metal budget at high redshifts.

The thermodynamic state of the filamentary gas causes a global feedback effect on the embedded galaxies by strangling the gas accretion (Finoguenov et al. 2003a; Oh \& Benson 2003). The resulting star-formation will proceed by consumption of the previously accumulated gas, in either quiescent mode as in the disk, or merger-induced bursts leading to formation of the spheroid (Somerville et al. 2001). Galaxy mergers are frequent inside galaxy groups (Kodama et al. 2001), but in a filament the infall of the gas will primarily be recorded in the star-formation history of the disk (Kennicutt et al. 1994).
A relevant observation could therefore shed light on the feedback epoch, which is crucial in understanding the relation between the X-ray filaments and OVI absorbers. The recent Sloan Digital Sky Survey (SDSS) discovery of passive spirals, in the same filament in front of Coma (Goto et al. 2003), is exactly what is expected from this strangulation process. The existence of these passive spirals lends further support to the association of the soft X-ray excess with the Coma filament. As passive spirals are starting to be found in the outskirts of many clusters, the presence of the soft X-ray excess might be a wide-spread phenomenon among the massive clusters, as indicated by first results on XMM-Newton REFLEX-DXL survey (Zhang et al. 2003).

\section{Conclusions}

We have discovered oxygen line emission associated with the outskirts of the Coma cluster. These data show that the previously observed soft X-ray excess in this cluster is due to thermal emission from a warm gas, possibly the long-sought WHIM. Typical parameters characterizing the emitting material are a temperature $\sim 0.2 \mathrm{keV}$, abundance $\sim 0.1$ solar, and density $\sim 50 f_{\text {baryon }} \rho_{\text {critical }}$. The thermodynamical state of the gas in the Coma filament reduces the star-formation rate of the embedded spiral galaxies, providing an explanation to the presence of the passive spirals in the SDSS survey in this region (Goto et al. 2003).

Acknowledgements. The paper is based on observations obtained with XMM-Newton, an ESA science mission with instruments and contributions directly funded by ESA Member States and the USA (NASA). The XMM-Newton project is supported by the Bundesministerium für Bildung und Forschung/Deutsches Zentrum für Luft- und Raumfahrt (BMFT/DLR), the Max-Planck Society and the Heidenhain-Stiftung, and also by PPARC, CEA, CNES, and ASI. AF acknowledges receiving the Max-Plank-Gesellschaft Fellowship. Authors thank Konrad Dennerl for applying the experimental bias correction software to the Coma-11 observation. AF profited from discussions with M. Freyberg, M. Geller, F. Nicastro, M. Markevitch, M. Arnaud, D. Neumann, A. Vikhlinin, and F. Miniati. AF would like to particularly mention a continuous support of this work by R. Lieu and his collaborators, M. Bonamente, J. Mittaz, and J. Kaastra. JPH thanks MPE for its hospitality during several extended visits. We acknowledge an anonymous referee for pointing out an importance of the detailed comparison between XMM-Newton and RASS.

\section{References}

Arnaud, M., Aghanim, N., Gastaud, R., et al. 2001, A\&A, 365, L67 Bonamente, M., Lieu, R., Joy, M., \& Nevalainen, J. 2002, ApJ, 576, 688

Bonamente, M., Joy, M., \& Lieu, R. 2003, ApJ, 585, 722

Briel, U. G., Henry, J. P., \& Böhringer, H. 1992, A\&A, 259, L31

Briel, U. G., \& Henry, J. P. 1995, A\&A, 302, 9

Briel, U. G., Henry, J. P., Lumb, D. H., et al. 2001, A\&A, 365, L60

Cen, R., \& Ostriker, J. P. 1999, ApJ, 514, 1

Colles, M., \& Dunn, A. M. 1996, ApJ, 458, 435

Davé, R., Cen, R., Ostriker, J. P., et al. 2001, ApJ, 552, 473

De Luca, A., \& Molendi, S. 2002, preprint [astro-ph/0202480]

Donnelly, R. H., Markevitch, M., Forman, W., et al. 1999, ApJ, 513, 690 
Dos Santos, S., \& Doré, O. 2002, A\&A, 383, 450

Evrard, A. E., \& Henry, J. P. 1991, ApJ, 383, 95

Fang, T., Marshall, H. L., Lee, J. C., Davis, D. S., \& Canizares, C. R. 2002, ApJ, 572, L127

Fang, T., Sembach, K. R., \& Canizares, C. R. 2003, ApJ, 586, L49

Finoguenov, A., Reiprich, T., \& Böhringer, H. 2001, A\&A, 368, 749

Finoguenov, A., Jones, C., Böhringer, H., \& Ponman, T. J. 2002, ApJ, 578,74

Finoguenov, A., Borgani, S., Tornatore, L., \& Böhringer, H. 2003a, A\&A, 398, L35

Finoguenov, A., Burkert, A., \& Böhringer, H. 2003b, ApJ, 594, 136

Freyberg, M. J., \& Breitschwerdt, D. 2002, Proc. of JENAM 2002, in press

Fukugita, M., Hogan, C. J., \& Peebles, P. J. E. 1998, ApJ, 503, 518

Goto, T., Okamura, S., Bernardi, M., et al. 2003, PASJ, submitted [astro-ph/0301303]

Haberl, F., Briel, U. G., Dennerl, K., \& Zavlin, V. E. 2002, to appear in Proceedings of "New Visions of the X-ray Universe in the XMM-Newton and Chandra Era", ESTEC, The Netherlands [astro-ph/0203235]

Hasinger, G., Altieri, B., Arnaud, M., et al. 2001, A\&A, 365, 45

Huchra, J. P., Geller, M. J., \& Corwin, H. G. Jr. 1995, ApJS, 99, 391

Jansen, F., Lumb, D., Altieri, B., et al. 2001, A\&A, 365, L1

Kaastra, J. S., Lieu, R., Tamura, T., Paerels, F. B. S., \& den Herder, J. W. 2003, A\&A, 397, 445

Kaiser, N. 1986, MNRAS, 222, 323

Kaiser, N. 1991, ApJ, 383, 104

Katayama, H., Takahashi, I., Ikebe, Y., Matsushita, K., \& Freyberg, M. J. 2002, A\&A, submitted [astro-ph/0210135]

Kennicutt, R., Tamblyn, P., \& Congdon, C. 1994, ApJ, 435, 22

Kodama, T., Smail, I., Nakata, F., Okamura, S., \& Bower, R. G. 2001, ApJ, 562, L9

Kull, A., \& Böhringer, H. 1999, A\&A, 341, 23

Lieu, R., Mittaz, J. P. D., Bowyer, S., et al. 1996, Science, 274, 1335

Lumb, D. H., Warwick, R. S., Page, M., \& De Luca, A. 2002, A\&A, 389,93
Lumb, D. H., Finoguenov, A., Saxton, R., et al. 2003, SPIE, 4851, 255 Markevitch, M. 1998, ApJ, 504, 27

Markevitch, M., Bautz, M. W., Biller, B., et al. 2003, ApJ, 583, 70

Mathur, S., Weinberg, D. H., \& Chen, X. 2003, ApJ, 582, 82

Miniati, F., Ryu, D., Kang, H., et al. 2000, ApJ, 542, 608

Nagamine, K., Fukugita, M., Cen, R., \& Ostriker, J. P. 2001, ApJ, 558, 497

Neumann, D. M., Arnaud, M., Gastaud, R., et al. 2001, A\&A, 365, L74

Nicastro, F., Zezas, A., Drake, J., et al. 2002, ApJ, 573, 157

Oh, S. P., \& Benson, A. J. 2003, MNRAS, 342, 664

Ponman, T. J., Cannon, D. B., \& Navarro, J. F. 1999, Nature, 397, 135

Ponman, T. J., Sanderson, A. J. R., \& Finoguenov, A. 2003, MNRAS, 343,331

Rasmussen, A., Kahn, S. M., \& Paerels, F. 2003, ASSL Conf. Proc., ed. J. L. Rosemberg, \& M. E. Putnam (Kluwer), 281, 109

Read, A. M. 2003, preprint [astro-ph/0212436]

Sanderson, A. J. R., Ponman, T. J., Finoguenov, A., Lloyd-Davies, E. J., \& Markevitch, M. 2003, MNRAS, 340, 989

Scharf, C., Donahue, M., Voit, G. M., Rosati, P., \& Postman, M. 2000, ApJ, 528, L73

Smith, R. K., Brickhouse, N. S., Liedahl, D. A., \& Raymond, J. C. 2001, ApJ, 556, L91

Snowden, S. L., Egger, R., Freyberg, M. J., et al. 1997, ApJ, 485, 125

Somerville, R. S., Primack, J. R., \& Faber, S. M. 2001, MNRAS, 320 , 504

Strüder, L., Briel, U. G., Dennerl, K., et al. 2001, A\&A, 365, L18

Tozzi, P., \& Norman, C. 2001, ApJ, 546, 63

Tripp, T. M., Savage, B. D., \& Jenkins, E. B. 2000, ApJ, 534, L1

Wang, Q. D., Connolly, A., \& Brunner, R. 1997, ApJ, 487, L13

Zappacosta, L., Mannucci, F., Maiolino, R., et al. 2002, A\&A, 394, 7

Zhang, Y.-Y., Finoguenov, A., Böhringer, H., et al. 2003, A\&A, submitted [astro-ph/0306115] 